\title{
Determinación de la precipitación efectiva en áreas agropecuarias de la provincia de Holguín
}

\author{
Determination of effective rainfall in agricultural areas of the province of Holguin \\ Juan Alejandro Villazón Gómez ${ }^{*}$, Pavel Noris Noris ${ }^{2}$, George Martín Gutiérrez ${ }^{3}$
}

\begin{abstract}
RESUMEN
La determinación de la precipitación efectiva constituye un elemento de suma importancia para una adecuada gestión de los recursos hídricos. El trabajo se desarrolló con el objetivo de determinar, mediante tres métodos, la precipitación efectiva mensual a partir de la precipitación probable en áreas agropecuarias de la provincia de Holguín. Se tomaron los datos de precipitaciones comprendidos entre los años 1975-2017, en cuatro estaciones meteorológicas (La Jíquima, Guaro, Pinares de Mayarí y Velasco). Las láminas diarias fueron totalizadas para los períodos mensuales correspondientes. La precipitación probable $(P p)$ se obtuvo para el 75 y el $90 \%$ de probabilidad de ocurrencia utilizándose el método probabilístico de distribución Gamma. A partir de la precipitación probable se determinó la precipitación efectiva por los métodos del porcentaje fijo, AGLW-FAO y USDA. Los métodos AGLWFAO y USDA arrojan valores de precipitación efectiva cercanos a los obtenidos por el método de porcentaje fijo para año "normal" $(P F 50)$ y "seco" (PF80). El comportamiento de la precipitación efectiva, independientemente del método utilizado, indica que resulta esencial tener en cuenta proyectos de riego para satisfacer las necesidades hídricas de los cultivos en los meses de invierno.

Palabras clave: hidrología, distribución Gamma, necesidades hídricas de los cultivos.
\end{abstract}

\begin{abstract}
The work developed with the objective to determine, by means of three methods, the effective monthly precipitation from the probable precipitation in agricultural areas of the province of Holguin. The data of precipitations understood between the yearstook 1975 2017 themselves, in four weather stations (La Jiquima, Guaro, Pinares de Mayarí and Velasco). The daily plates were totalized for the monthly corresponding periods. The probable precipitation (Pp) was once funny remark being used was gotten from for the 75 and $90 \%$ of probability the probabilistic method of distribution Gamma. From the probable precipitation determined him the effective precipitation for the methods of the fixed percentage, AGLW-FAO and USDA. Methods AGLW-FAO and USDA throw close values of effective precipitation at the obtained by the method of fixed percentage for "normal" (PF50) and "dry" year (PF80). The behavior of the effective precipitation, regardless of the used method, indicate that it proves to be necessary to take in to account projects of irrigation to satisfy the agricultural hydric requirements in the months of winter.

Keywords: hydrology, Gamma distribution, agricultural hydric requirements.
\end{abstract}

\section{Introducción}

El agua en el suelo constituye un recurso fundamental para las actividades agrícolas, por lo que su disponibilidad resulta esencial para el crecimiento y desarrollo vegetal (Syperreck et al., 2008). En la medida en que el agua se vuelve cada vez más escasa y la necesidad se torna más apremiante, es indispensable realizar mediciones avanzadas y técnicas de evaluación del manejo de los recursos hídricos. Alrededor del 17\% de la superficie cultivada del mundo está irrigada y aporta más de un tercio de la producción total mundial de alimentos. En todo el mundo, la agricultura de regadío utiliza la mayor parte de las extracciones de agua de los suministros de agua superficial y

1 Universidad de Holguín. Sede José de la Luz y Caballero, Facultad de Ciencias Naturales y Agropecuarias, Centro de Estudios para Agroecosistemas Áridos (CEAAR), Holguín, Cuba.

2 Centro Provincial de Meteorología, Delegación Provincial del Ministerio de Ciencias, Tecnología y Medio Ambiente (CITMA), Holguín, Cuba.

3 Estación Provincial de Investigaciones de la Caña de Azúcar (EPICA) de Holguín.

* Autor por correspondencia: villazon@uho.edu.cu.

Fecha de Recepción: 3 de Septiembre, 2019.

Fecha de Aceptación: 21 de Octubre, 2020 
subterránea. Por lo tanto, la cuantificación precisa del uso del agua en los cultivos es crucial para una mejor gestión y asignación de los recursos hídricos (Wane y Nagdeve, 2014).

La caracterización de las precipitaciones es significativa para muchos tipos de estudios hidrológicos, principalmente para aquellos que tienen como objetivo estimar el volumen de flujo y la descarga máxima de escorrentía superficial y las pérdidas por erosión (Back, 2011). No toda la lluvia puede ser aprovechada por los cultivos: parte de ella se pierde por escorrentía, percolación profunda o evaporación (Patel et al., 2017). De esta manera, la precipitación efectiva es la parte de la lluvia caída en un sitio determinado que puede ser aprovechada directa o indirectamente por los cultivos establecidos en el área (Dastane, 1974) y es posible calcularla por diferentes métodos (Kokkonen et al., 2004; Tsai et al., 2005; Rahman et al., 2008; Ganesh et al., 2015; Khandelwal y Dhiman, 2015).

Es importante en las decisiones de programación de riego. Se utiliza, además, para diseñar nuevos sistemas de riego y es un factor guía para planificar las prácticas de producción de cultivos. Las precipitaciones efectivas dependen del estado de humedad del suelo (o del régimen de riego), las características del cultivo, las propiedades del suelo y del comportamiento de la lluvia. Para condiciones específicas del suelo, es particularmente dependiente de la evapotranspiración y la lluvia (Ali y Mubarak, 2017).

El objetivo de este trabajo es determinar, mediante tres métodos, la precipitación efectiva mensual a partir de la precipitación probable en áreas agropecuarias de la provincia de Holguín.

\section{Materiales y métodos}

El trabajo se desarrolló con los datos mensuales de precipitaciones tomados en las estaciones meteorológicas de La Jíquima, Guaro, Pinares de Mayarí y Velasco (Tabla 1), pertenecientes al Instituto de Meteorología en la provincia de Holguín, Cuba, entre los años 1975-2017.

Las estaciones meteorológicas se encuentran ubicadas en áreas donde predominan suelos Haplusterts, Haplustepts, Eutrudepts, Eutrudoxs, Haplustolls y Haprendolls (Soil Survey Staff, 2010), dedicados fundamentalmente al cultivo de la caña de azúcar, arroz, hortalizas, plátano, frijol, frutales, pastos naturales, y a fines forestales. El clima de la región se considera tropical lluvioso, presenta inviernos secos y veranos húmedos, registrado como $A w$, según la clasificación de Köppen.

Las láminas diarias fueron totalizadas para los períodos mensuales correspondientes. La precipitación probable $(P p)$ se obtuvo para el 75 y el $90 \%$ de probabilidad de ocurrencia (Sampaio et al., 2000; Silva et al., 2010) utilizándose el método probabilístico de distribución Gamma.

La función de densidad de probabilidad se define como:

$$
f(x)=\frac{1}{\beta^{a} \Gamma(\alpha)} x^{\alpha-1} e^{-\frac{x}{\beta}} x \geq 0
$$

Donde $\alpha$ es el parámetro de forma (adimensional), $\beta$ es el parámetro de escala $(\mathrm{mm}), e$ es la base del logaritmo neperiano, $x$ es la precipitación $(\mathrm{mm})$ y $\Gamma$ es la función del mismo nombre. Las estimaciones de los parámetros $\alpha$ y $\beta$ fueron realizadas por el método de máxima verosimilitud mediante las expresiones siguientes:

$$
\begin{gathered}
\alpha=\frac{1}{4 \mathrm{~A}}\left(1+\sqrt{\frac{4 \mathrm{~A}}{3}}\right) \\
\beta=\frac{\bar{X}}{\alpha}
\end{gathered}
$$

Siendo:

$$
\begin{gathered}
\mathrm{A}=\ln \overline{\mathrm{X}}-\mathrm{X}_{\mathrm{g}} \\
\bar{X}=\frac{1}{\mathrm{~N}} \sum_{\mathrm{i}=1}^{\mathrm{N}} \mathrm{X}_{\mathrm{i}} \\
\mathrm{X}_{\mathrm{g}}=\frac{1}{\mathrm{~N}} \sum_{\mathrm{i}=1}^{\mathrm{N}} \ln \left(\mathrm{x}_{\mathrm{i}}\right)
\end{gathered}
$$

Donde $\overline{\mathrm{X}}$ es la media aritmética, $\mathrm{X}_{\mathrm{g}}$ es la media geométrica y $\mathrm{N}$ es el número de meses observados.

La función Gamma se determina a partir de (1):

$$
\Gamma(\alpha)=\int_{0}^{\infty} x^{a-1} e^{-x} d(x)
$$

La distribución acumulada Gamma se describe como:

$$
F(x)=\frac{1}{\beta^{a} \Gamma(a)} \int_{0}^{x} x^{a-1} e^{-\frac{x}{\beta}} d(x)
$$


Tabla 1. Ubicación geográfica y características climáticas de las estaciones meteorológicas.

\begin{tabular}{|c|c|c|c|c|c|}
\hline \multirow{2}{*}{ Estaciones meteorológicas } & \multicolumn{2}{|c|}{ Coordenadas } & \multirow{2}{*}{$\begin{array}{c}\text { Precipitación } \\
\text { anual (mm) }\end{array}$} & \multirow{2}{*}{$\begin{array}{l}\text { Temperatura } \\
\text { media }\left({ }^{\circ} \mathrm{C}\right)\end{array}$} & \multirow{2}{*}{$\begin{array}{c}\text { Altitud } \\
\text { (msnmm) }\end{array}$} \\
\hline & Latitud N & Longitud W & & & \\
\hline La Jíquima & $20^{\circ} 59^{\prime} 18^{\prime \prime}$ & $76^{\circ} 32^{\prime} 23^{\prime \prime}$ & $1.137,5$ & 25,4 & 106,77 \\
\hline Guaro & $20^{\circ} 40^{\prime} 21^{\prime \prime}$ & $75^{\circ} 46^{\prime} 57^{\prime \prime}$ & $1.067,6$ & 25,6 & 20,96 \\
\hline Pinares de Mayarí & $20^{\circ} 29^{\prime} 14^{\prime \prime}$ & $75^{\circ} 47^{\prime} 26^{\prime \prime}$ & $1.686,4$ & 21,6 & 647,04 \\
\hline Velasco & $21^{\circ} 04^{\prime} 03^{\prime \prime}$ & $76^{\circ} 20^{\prime} 42^{\prime \prime}$ & $1.221,8$ & 25,7 & 61,00 \\
\hline
\end{tabular}

msnmm: metros sobre el nivel medio del mar.

Los resultados de la estimación de la precipitación probable mensual (para un 75 y un $90 \%$ de ocurrencia) se muestran en la Tabla 2.

A partir de la precipitación probable se realizó la estimación de la precipitación efectiva $(P e)$ a través de tres métodos: porcentaje fijo, AGLWFAO y USDA (Sampaio et al., 2000; Silva et al., 2010; Wane y Nagdeve, 2014).

El método de porcentaje fijo es definido como la precipitación efectiva con un porcentaje relativo a la precipitación probable. De esta forma, para un $20 \%$ de la precipitación probable se considera un año húmedo (PF20), para un 50\% se contempla un año normal $(P F 50)$ y para un $80 \%$ representa un año seco $(P F 80)$. De esta manera, cuanto más húmedo es el año, menor es el porcentaje de precipitaciones que, al quedar retenido en el suelo, se encuentra disponible para las plantas.

El método de AGLW-FAO fue desarrollado para climas de áridos a subhúmedos. Las ecuaciones empíricas para estimar la precipitación efectiva
$(P e)$, que tienen en cuenta las pérdidas de agua por escorrentía y percolación, son:

$$
\begin{array}{rr}
\mathrm{P}_{\mathrm{e}}=0,6 * \mathrm{P}_{\rho}-10 & \mathrm{P}_{\rho} \leq 70 \mathrm{~mm} \\
\mathrm{P}_{\mathrm{e}}=0,8 * \mathrm{P}_{\rho}-24 & \mathrm{P}_{\rho} \leq 70 \mathrm{~mm}
\end{array}
$$

El método USDA fue desarrollado a partir de balances hídricos, relacionándose los ingresos de agua por precipitación con las salidas por escurrimiento superficial y percolación, así como el agua retenida en la zona radicular para varios cultivos.

$$
\begin{gathered}
\mathrm{P}_{\mathrm{e}}=\frac{\mathrm{P}_{\rho} *\left(125-0,2 * \mathrm{P}_{\rho}\right)}{125} \mathrm{P}_{\rho} \leq 250 \mathrm{~mm} \\
\mathrm{P}_{\mathrm{e}}=125+0,1 * \mathrm{P}_{\rho} \mathrm{P}_{\rho}>250 \mathrm{~mm}
\end{gathered}
$$

\section{Resultados y Discusión}

\begin{tabular}{|c|c|c|c|c|c|c|c|c|c|c|c|c|}
\hline & ene & feb & mar & abr & may & jun & jul & ago & Sep & oct & nov & dic \\
\hline \multicolumn{13}{|c|}{ La Jíquima } \\
\hline $75 \%$ & 12,1 & 4,6 & 8,7 & 23,5 & 107,7 & 94,0 & 78,4 & 95,3 & 107,9 & 63,2 & 23,0 & 16,1 \\
\hline $90 \%$ & 3,4 & 2,8 & 1,6 & 4,6 & 58,0 & 62,8 & 36,7 & 72,1 & 82,1 & 36,2 & 10,4 & 10,6 \\
\hline \multicolumn{13}{|c|}{ Guaro } \\
\hline $75 \%$ & 27,8 & 18,8 & 21,7 & 32,1 & 46,1 & 52,4 & 23,8 & 44,9 & 56,5 & 86,3 & 68,1 & 50,3 \\
\hline $90 \%$ & 20,0 & 6,3 & 12,2 & 15,3 & 39,1 & 36,4 & 6,4 & 35,9 & 47,4 & 55,3 & 31,5 & 23,8 \\
\hline \multicolumn{13}{|c|}{ Pinares de Mayarí } \\
\hline $75 \%$ & 20,1 & 34,0 & 31,3 & 64,1 & 180,0 & 159,6 & 106,8 & 127,0 & 166,1 & 129,6 & 56,0 & 20,6 \\
\hline $90 \%$ & 9,1 & 10,2 & 11,5 & 20,1 & 132,1 & 138,7 & 86,8 & 110,3 & 139,5 & 104,7 & 28,4 & 14,6 \\
\hline \multicolumn{13}{|c|}{ Velasco } \\
\hline $75 \%$ & 14,4 & 9,5 & 10,7 & 23,8 & 114,5 & 93,6 & 65,0 & 88,6 & 111,4 & 68,4 & 25,5 & 21,9 \\
\hline $90 \%$ & 4,6 & 3,1 & 1,6 & 6,6 & 61,7 & 66,0 & 29,8 & 72,1 & 46,7 & 37,8 & 21,5 & 16,1 \\
\hline
\end{tabular}

La Figura 1 muestra los resultados de la lluvia probable obtenidos a partir del método del

Tabla 2. Estimación de la precipitación probable mensual para el 75 y el 90\% de ocurrencia. 

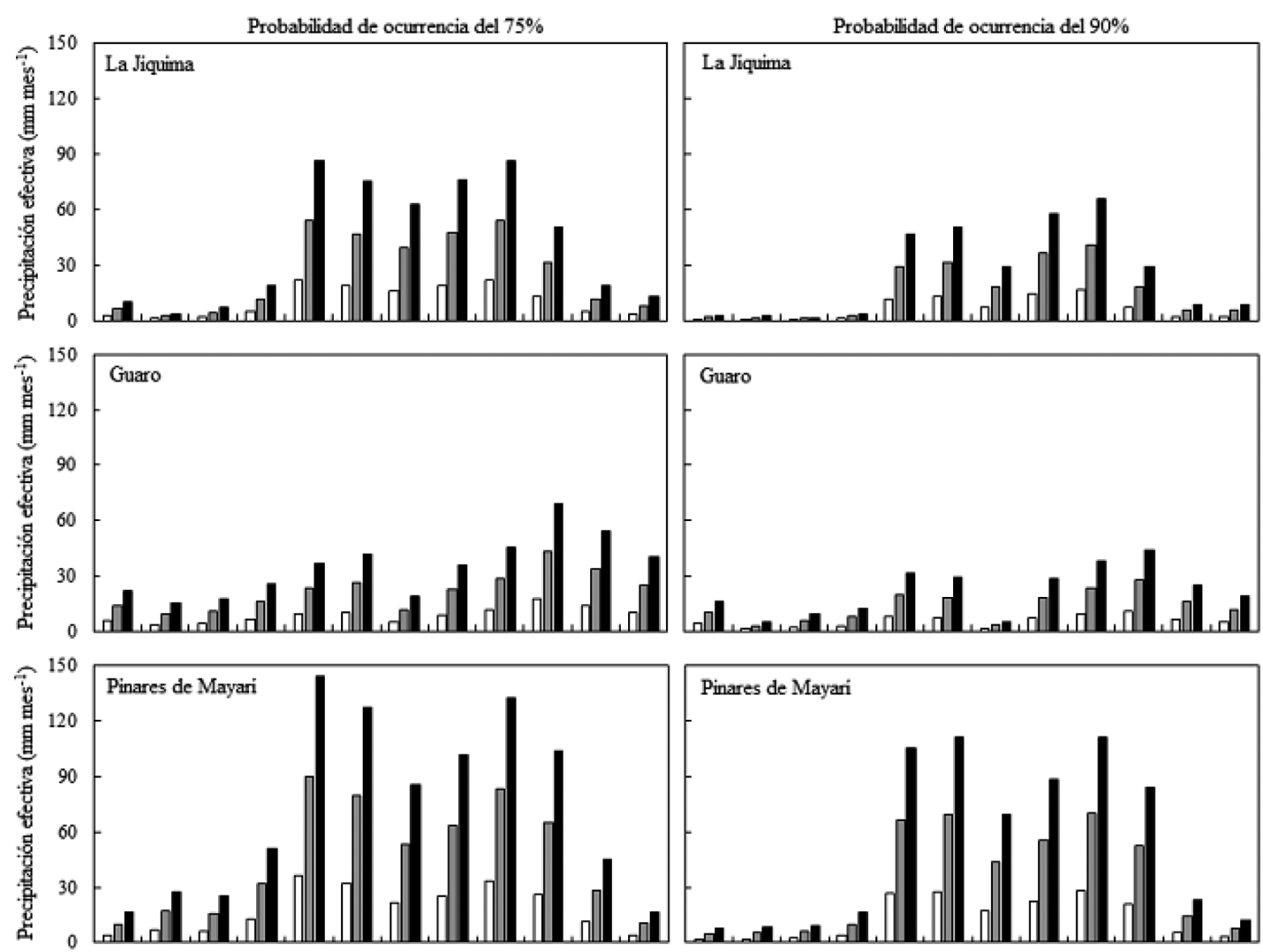

Pinares de Mayari
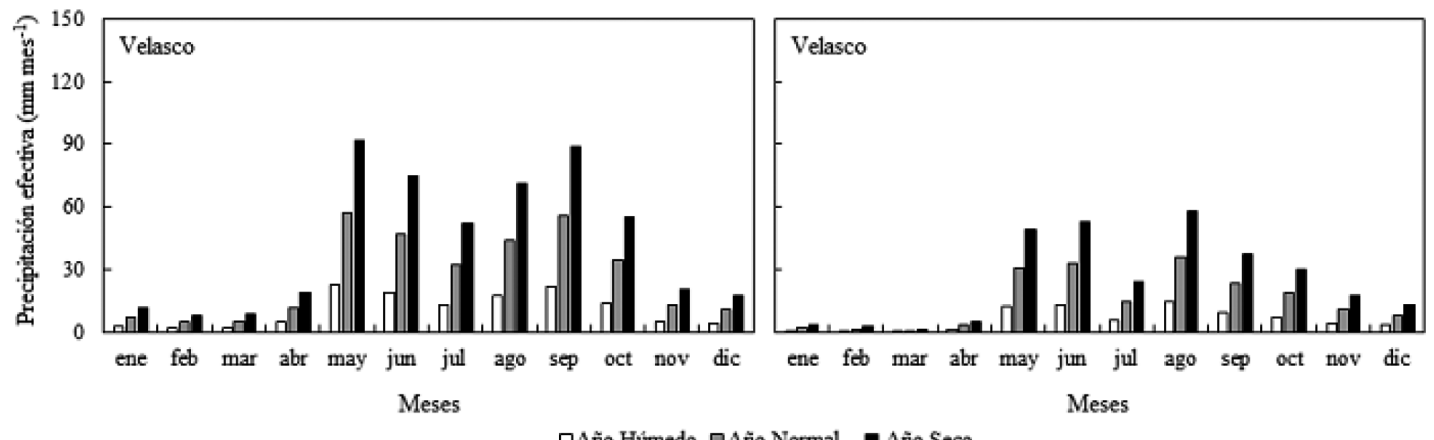

Figura 1. Comportamiento de la precipitación probable mensual por el método de porcentaje fijo.

porcentaje fijo. Se observa que los menores valores de precipitación efectiva se determinaron en los años "húmedos" y los mayores en los años "secos". Al realizar un estudio en diferentes zonas climáticas del sureste de Bangladesh, durante el período 2006-2007, Rahman et al. (2008) encontraron que la precipitación efectiva era directamente proporcional al consumo hídrico de los cultivos e inversamente proporcional a la cantidad e intensidad de precipitaciones totales caídas.
Resultan notables los bajos valores de precipitación efectiva, en todas las estaciones meteorológicas, en los meses de invierno, sobre todo para un $90 \%$ de probabilidad de ocurrencia. Esto indica la necesidad, en caso de no contar con los recursos indispensables para establecer sistemas de riego durante ese período, de plantar cultivos o variedades de cultivos tolerantes a la sequía.

Los valores más altos por estación meteorológica para los tres porcentajes fijos (PF20, PF50 y 
PF80) se encontraron en Pinares de Mayarí. En dicha área, dedicada fundamentalmente a fines forestales debido a la baja fertilidad química de los suelos (clasificados dentro de los subgrupos Eutrudox típico, Eutrudoxpetroférrico y Eutrudox húmico), resulta importante si se tiene en cuenta el predominio de formas de relieve con determinada pendiente. A ello se suma la propensión de estos suelos a erosionarse cuando carecen de cobertura vegetal. Por lo tanto, una elevada precipitación efectiva, unida al drenaje excesivo característico de estos suelos, atenúa los riesgos de erosión.

La Figura 2 muestra la precipitación efectiva a partir del método AGLW-FAO. Este arroja valores de precipitación efectiva por encima de los obtenidos con el método PF50 (año "normal") durante los meses de mayores precipitaciones: mayo-septiembre para La Jíquima, octubre para Guaro, mayo-octubre para Pinares de Mayarí y mayo-junio y septiembre para Velasco.
La Figura 3 muestra los valores de precipitación efectiva a partir del método USDA. Al comparar las precipitaciones efectivas obtenidas por el método USDA y por el PF80 (año "seco"), se observa que con el primero invariablemente se logran valores de precipitación efectiva más altos, siempre y cuando la precipitación probable (para un $75 \%$ ) no supere los $159 \mathrm{~mm}$.

Al comparar el método USDA con el AGLWFAO se observa que el primero siempre arroja valores de precipitación probable más altos que el segundo, y que esta diferencia es más notable en los meses húmedos de verano que en los meses correspondientes al invierno seco.

De forma general, puede apreciarse que los métodos AGLW-FAO y USDA poseen valores semejantes a los obtenidos por el método de porcentaje fijo para un año "normal" y "seco", respectivamente. Esto hace indispensable, en los meses de invierno, la utilización del riego para suplir las necesidades hídricas de los cultivos.

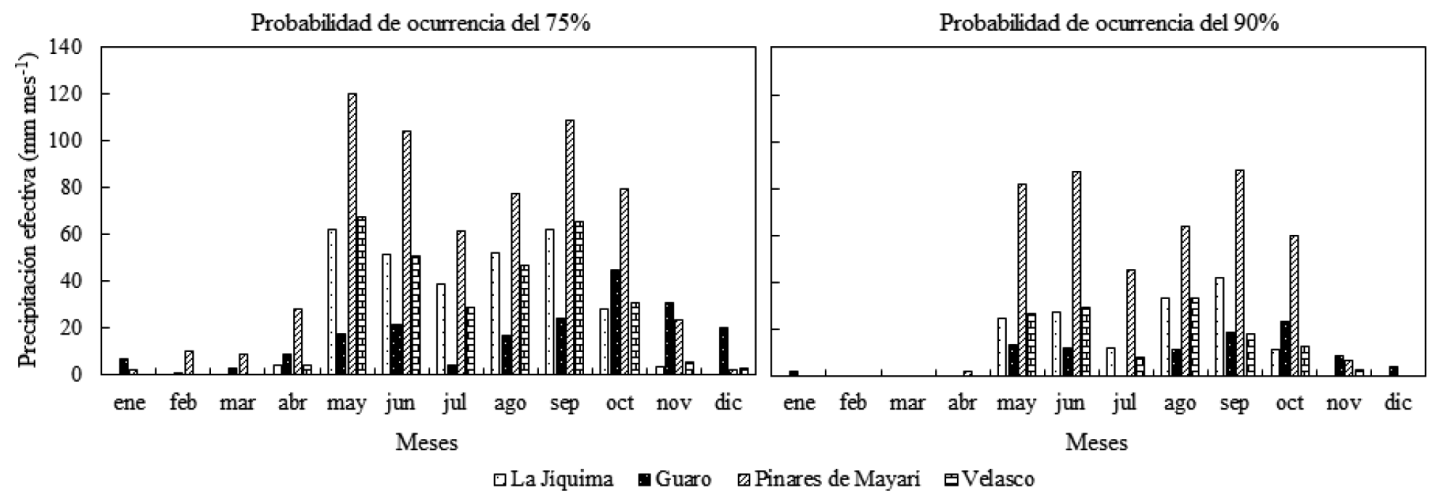

Figura 2. Comportamiento de la precipitación probable mensual por el método AGWL-FAO.

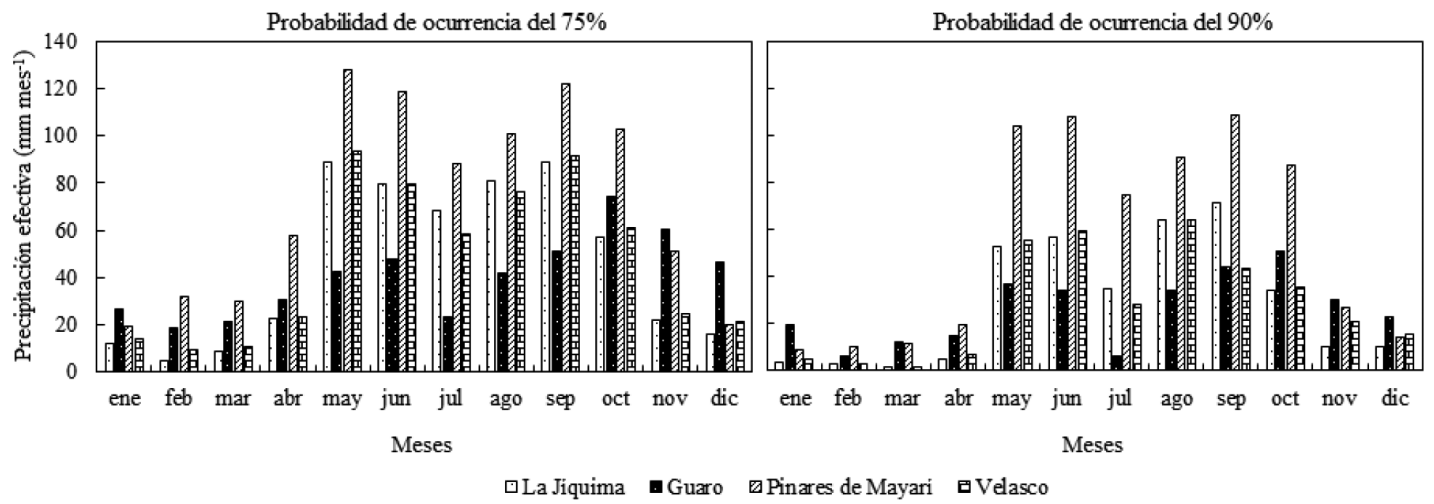

Figura 3. Comportamiento de la precipitación probable mensual por el método USDA. 
Resultados similares obtuvieron Sampaio et al. (2000), al aplicar estos tres métodos al estudio del comportamiento de la lluvia efectiva en la región de Lavras, estado de Minas Gerais (Brasil), con la recomendación de utilizar los valores encontrados a partir de la lluvia probable para un $90 \%$ en proyectos de riego, en cultivos sensibles a los efectos del estrés hídrico o en los de gran importancia económica, y dejar para el resto de los cultivos los resultados logrados a partir de la precipitación probable para el $75 \%$ de probabilidad de ocurrencia.

\section{Conclusiones}

Los métodos AGLW-FAO y USDA arrojan valores de precipitación efectiva cercanos a los obtenidos por el método de porcentaje fijo para año "normal" (PF50) y "seco" (PF80).

El comportamiento de la precipitación efectiva, independientemente del método utilizado, indica que resulta esencial tener en cuenta proyectos de riego para satisfacer las necesidades hídricas de los cultivos en los meses de invierno.

\section{Literatura Citada}

Ali, M.H.; Mubarak, S.

2017. Effective rainfall calculation methods for field crops: an overview, analysis and new formulation. Asian Research Journal of Agriculture, 7(1): 1-12.

Back, Á.J.

2011. Time distribution of heavy rainfall events in Urussanga, Santa Catarina State, Brazil. Acta Scientiarum. Agronomy, 33(4): 583-588.

Dastane, N.G.

1974. Effective rainfall in irrigated agriculture. F.A.O. irrigation and Drainage paper 25, Food and Agriculture Organization of the United Nations. Roma, Italia. 68 p.

Ganesh Babu, R.; Ravi Babu, G.; Hema Kumar, H.V.

2015. Estimation of crop water requirement, effective rainfall and irrigation water requirement for vegetable crops using CROPWAT. International Journal of Agricultural Engineering, 8(1): 15-20.

Khandelwal, S.S.; Dhiman, S.D.

2015. Irrigation water requirements of different crops in Limbasi branch canal command area of Gujarat. Journal of Agrometeorology, 17(1): 114-117.

Kokkonen, T.; Koivusalo, H.; Karvonen, T.; Croke, B.; Jakeman, A.

2004. Exploring stream flow response to effective rainfall across event magnitude escale. Hydrological Processes, 18: 1467-1486.

Patel, A.; Sharda, R.; Patel, S.; Meena, P.

2017. Reference evapotranspiration estimation using CROPWAT model at Ludhiana district (Punjab). International Journal of Science, Environment and Technology, 6(1): 620-629.
Rahman, M.M.; Islam, M.O.; Hasanuzzaman, M.

2008. Study of effective rainfall for irrigated agriculture in south-eastern part of Bangladesh. World Journal of Agricultural Sciences, 4(4): 453-457.

Sampaio, S.C.; MetriCorrêa; M.; Vilas Bôas, M.A.; Coutinho de Oliveira, L.F.

2000. Estudo da precipitação efetiva para o município de Lavras, MG. Revista Brasileira de Engenharia Agrícola e Ambiental, 4(2): 210-213.

Silva, Ana P.N. da; Lima, F.J.L. de; Silva, A.O. da; Moura, G.B. de A.

2010. Valores efetivos de precipitação pluvial para manejo da irrigação na cana-de-açúcar em Goiana, Pernambuco. Revista Brasileira de Ciências Agrárias, 5(4): 585-591.

Soil Survey Staff.

2010. Keys to Soil Taxonomy. USDA, 338 p.

Syperreck, Vera Lucia Greco; Klosowski, E.S.; Greco, M.; Furlanetto, C.

2008. Avaliação de desempenho de métodos para estimativas de evapotranspiração de referência para a região de Palotina, Estado do Paraná. Acta Scientiarum. Agronomy, 30 (suplemento especial): 603-609.

Tsai; S.M.; Chen, Sh.; Wang, H.Y.

2005. A study on the practical model of planned effective rainfall for paddy fields in Taiwan. Journal of Marine Science and Technology, 13(2): 73-82.

Wane, S.S.; Nagdeve, M.B.

2014. Estimation of evapotranspiration and effective rainfall using CROPWAT. International Journal of Agricultural Engineering, 7(1): 23-26. 